\title{
Mobile Phone cards recharge using OCR (Applied on android platform)
}

\author{
Dr. Amr Mahmoud El Maghraby \\ High Institute for Computers and Information Systems \\ Al- Shorouk Academy \\ Cairo - Egypt \\ e.mail: dr.amr.elmaghraby@ sha.edu.eg
}

\begin{abstract}
This paper focuses on finding a new easy and effective approach to recharging the phone cards utilizing the innovation in our hand as mobile camera and OCR (Optical character recognition) technology. The main advantages of the proposed solution are made up of a combination of hardware and software that is used to convert physical recharge card into machine-readable text to saved time, decreased errors and minimized effort. According to the study of systems development life cycle (SDLC) new demands create new ideas and invent new technology. This Paper can be worthy in real life experience because recharging our mobile cards is one of the activities that nearly all the people do. So instead of entering the numbers manually, we present a new way in recharging mobile cards automatically by detect pin number portion from mobile recharge cards by image processing detection technique with the Technology of OCR for recharge processes. After the detection, and extracting processing the OCR text that is given within the recharge card it sends request to the respective mobile operator for recharging balance. Our main idea is to build an android based application replace the typing of pin numbers with a few seconds snap-shot recharge, making the recharging process much easier and quicker. This app eliminates all the troubles we face in dialing and enter the correct recharge number effectively from the first run through. We will use the machine learning and specifically the optical character recognition in order to make recharging much easier and prevent errors from occurring. We have built an application based on android cell phone making the recharging process much easier and quicker
\end{abstract}

Key Words: Image processing, OCR technology, Android Studio IDE, identification, verification process, identity verification, Authentication, recharge cards, Google mobile vision, OCR based snapshot recharge; 
Mobile Phone cards recharge using OCR .. (Applied on android platform)

\section{INTRODUCTION}

Innovative technology is rapidly changing our world. Now the most smartphone devices across brands have common technology. Today smartphone is an integrated computer with cellular telephone that included hardware like processor, embedded memory, a virtual or thumb keyboard, touchscreen, Wi-Fi, a digital camera typically with video capability, GPS global positioning system, Bluetooth capability, mic, headphones, integrated storage like internal memory and SD card that are mostly external and the two main category of software like System software is used for operating computer hardware and application software features .However, still the camera and its application are areas where innovation continues.

In 2020 the number of mobile phone users is forecast to reach 4.78 billion. In 2019 the number of mobile phone users is forecast to reach 4.68 billion.[1] Our application is targeting all the smart mobile phone users there have to be a simple and easy way to make the user recharge their mobile cards.

\section{General problem}

Generally, this study is used to saved time, decreased errors and minimized effort for recharge mobile service balance using scratch card.

1. Mobile phone operators generate scratch card (with unique id from 1xxxxxx9yyyyyy) for a particular plan (i.e 100,200,500,1000).

2. This unique Ids are hidden first (have to scratch) and then you apply that code using Unstructured Supplementary Service Data (USSD), sometimes referred to as "Quick Codes" or "Feature codes", is a communications protocol used by GSM cellular telephones to communicate with the mobile network operator's computers. [2]

3. This unique code goes to system (IN node), which checks whether this unique code has been used before or not. If not used, it credits the balance in your database Service Data Point (SDP) node in the intelligent network Global System for Mobile Communications (GSM) services which is responsible for providing subscriber information and make that unique code inactive for future use.[3]

4. Once credit the balance successfully, you can use the service as you like. This all process takes time. Our proposed solution use to make this process fast. Phone scratch card manufacturer offers an extensive range recharge scratch cards consisting of composite layering \& fraud-resistant security scratch panel, alongside certified ISO certified data controlling. Recharge cards contain a highly secure scratch pad hiding the pin code based on High resolution offset printing on both 
card sides. They can be produced based on different materials like paper (cardboard) and Polyvinyl Chloride (PVC) in compliance with ISO ID-1 (85x54mm) format.

Prepaid billing process involves the following important steps along with account information gathering and updating account after the call completed.

- Authenticating - Authentication is the process of verifying that a user is who he or she claims to be. The user supplies a user ID and an authentication credential, such as a password. The system accepts these as inputs and verifies that the user is valid and has access to the system. If the recharge card uses a 16 digit combination. The no of combinations of 16 digits in a recharge coupon is $9^{*} 10^{\wedge} 15$, In which all combinations may not exist, also after more than 3 failed attempt ,your number will be blocked and you have to contact customer care to unblock it, Thus the chances of getting a recharge with random guessing is almost nullified.

- Authorizing - Authorization is the process of verifying what an authenticated user allowed to do. Generally, Remote Access Dial in User Server (RADIUS) protocol is used to limit access to the system to registered and authorized customers. The RADIUS server checks that the information is correct using authentication schemes such as Password Authentication Protocol (PAP), Challenge-Handshake Authentication Protocol (CHAP)or Extensible Authentication Protocol (EAP). [3]

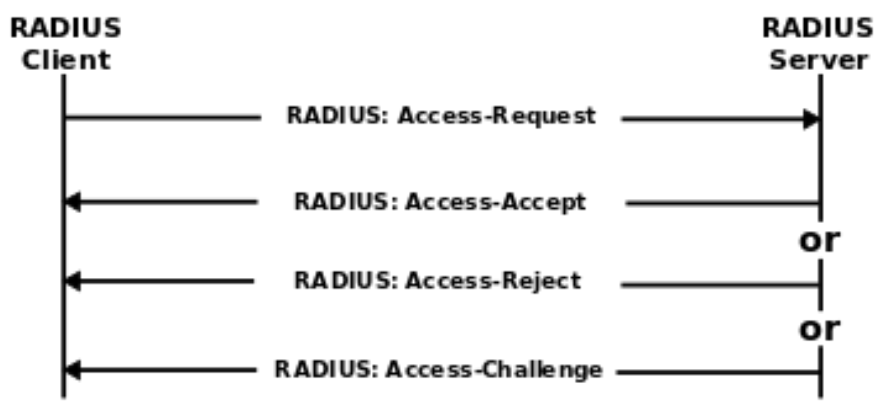

Figure 1: RADIUS Authentication and Authorization Flow

- Providing advice of charge (AOC): Gives information about the actual cost of the call either prior to or after the event. AOC provides the ability of a telecommunications system to advice of the actual costs of the event either prior to or after the occurrence of the event. 
The main problem is that when we want to recharge mobile phone, we have to enter the recharging code manually. The recharging code consist of 15 digits in addition to the quick code which consists of 5 digits so the overall digits will be 20 and in some other recharging cards the code consists of 16 digit or 15 digit depend on Mobile Network Operator (MNO) Vodafone 16 digit, other operator 15 digit so overall will be 21 ,so there is a great possibilities that you might enter one or more than one digit wrong by mistake and in in this case the company will send you message saying invalid code, and we will have to enter all the 20 digits or 21 digits all over again from the beginning and this can be so frustrating sometimes.

There are other cases for example:

1- You might have like 5 recharging cards that you want to recharge all these cards, so for each card you will have to repeats the process of enter the code and this will take time.

2- Some users can not clearly see the numbers because they are too small in some cards, this might result that they enter the wrong numbers.

However, with this idea and our practical application this will no longer be the case.

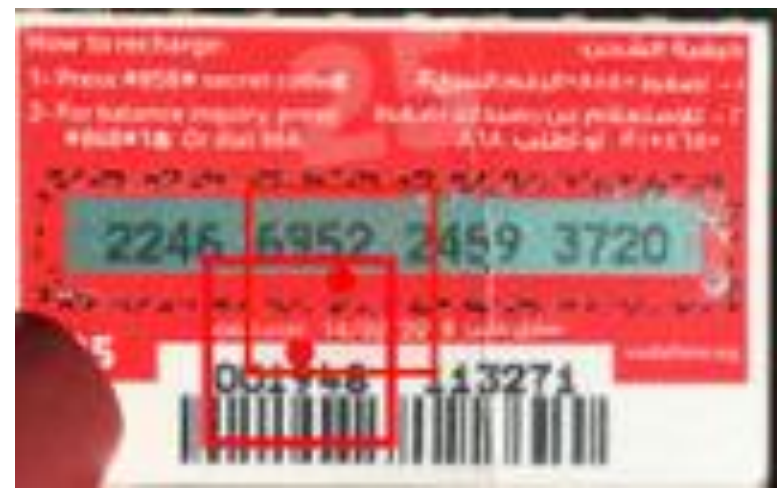

Figure 2: The total number of recharging codes consist of 16 number

\section{Practical Problems (Use Case Problems)}

-The first problem is that the application will need to know the type of the user's sim or what Mobile Network Operator (MNO) he belongs to in order to be able to 
automatically enter the quick code which differs from one mobile network carrier to another.

-The second problem is the degree of accuracy of the optical character recognition.

The aggregate aim of this applied scientific research is to recharge mobile in an easy way. In more detail: -

- The aim is to develop a mobile application based on optical Character recognition to help you recharge your mobile card by capturing the code only.

- Develop a mobile application that will target nearly all the smart phone users.

- To eliminate errors when recharging phone using Scratch Card.

- Make you able to recharge your card correctly from the first time.

- Save time and make mobile recharging easier and faster by only

Capturing the code in the card without having to enter code manually.

\section{Background}

Presently before going into the subtleties of the undertaking. We should have some opportunity to talk about the camera , optical character recognition and Similar application The core of our proposal is based on Digital cameras and Offline Mobile Optical Character Recognition (MOCR)

\subsection{Digital cameras}

Digital cameras are commonly found on mobile and it is different from analog film cameras completely in technology, first of all that they do not use film. Digital camera captures the image on charge coupled device (CCD)/ complementary metal-oxide-semiconductor (CMOS) sensor which captures the light and transforms it into a picture sensor and stores it on digital memory cards or internal storage or directly to the cloud and the images can be viewed immediately. in addition to image capture circuitry, is capable of extracting application-specific information from the captured images, along with generating event descriptions or making decisions that are used in an intelligent and automated system the following are the major Advantages: and Disadvantage in Smartphone cameras.[4]

Advantages: Smartphone cameras are small, light and easy to carry you don't have to carry them in a specialized bag. Smartphones allow zooming in or out. 
Mobile Phone cards recharge using OCR .. (Applied on android platform)

Disadvantage: Smartphone cameras have limited manual control options. We can't control lighting, aperture, depth of field, shutter speed.

\subsection{OCR technology}

OCR (optical character recognition) is the use of technology to distinguish printed or handwritten text characters inside digital images. Raymond Kurzweil who is an American computer scientist. He invented the first optical character recognition (OCR) software for transforming the written word into editable data, in order to help the blind people by making them a program which will read to them the text out loud. The field of OCR is divided into two parts, one is recognition of machine printed characters and the second is recognition of handwritten characters.

The Mobile Optical Character Recognition (MOCR) applications on regular smartphones holds great promise to access printed information. The proposed solution focus on recognition of machine printed characters and the use of offline and online optical character recognition (OCR) process for converting numerals, letters, and symbols in recharge card into machine readable character streams. The difference between offline character and online character is that Offline character recognition works on scanned images and online character recognition takes the input at run time.

\subsection{How the system applies Mobile Optical Character Recognition (MOCR)}

The goals and objectives of proposed system are to improve the quality of input image so that the OCR engine gives an accurate output.

\subsubsection{Preprocessing}

We Use the following image processing operation to improve the quality of your input image. Optical Character Recognition (OCR) systems often generate errors for images with noise or with low scanning resolution. Mostly OCR engine give an accurate output of the image which has 300 DPI (dots per inch). 


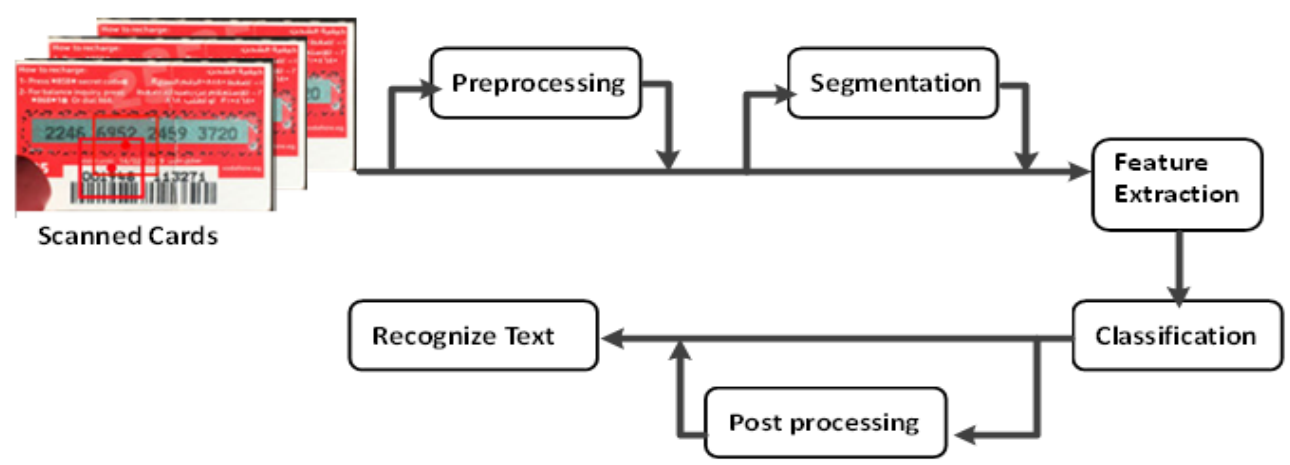

Fig 3 : Image processing block diagram operation

\section{1- Detect the text block with skew in the image.}

A Skewed image is defined as a document image which is not straight.

Skewed images directly impact the line segmentation of OCR engine which reduces its accuracy. We need to process the following steps to correct text skew.[5]

- Detect the text block with skew in the recharge card.

- Calculate the angle of rotation.

- Rotating the recharge card to correct the skew.
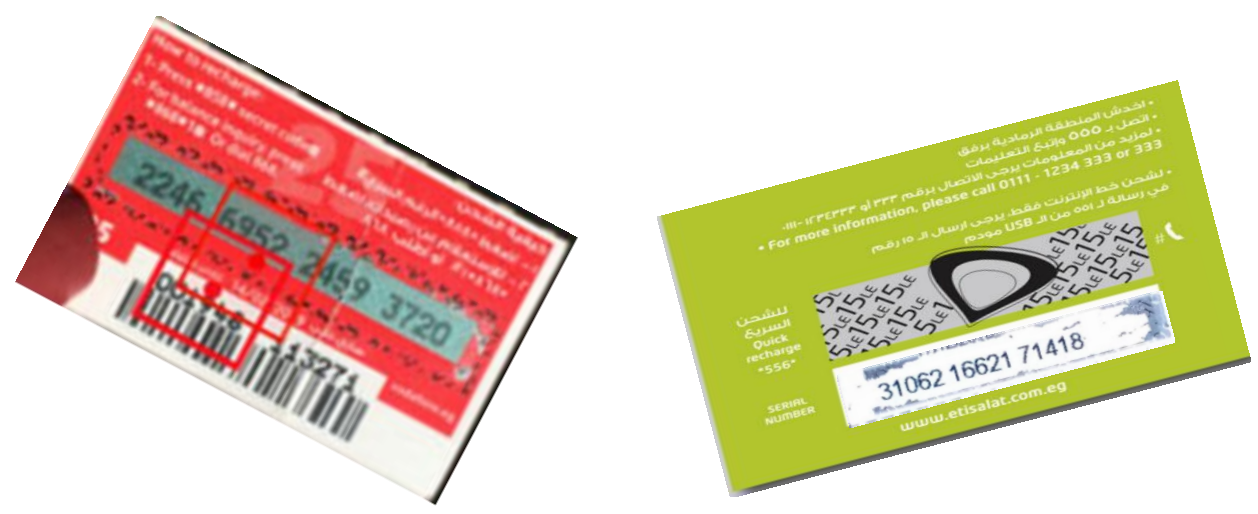

Fig 4 : Skewed Image $\quad-2$

\section{3- OCR binarization}

Some documents are difficult or downright impossible to recognize when scanned in black-and-white. In the "bilevel" mode, every pixel in the image is either white 
Mobile Phone cards recharge using OCR .. (Applied on android platform)

or black. When the text is printed on a colored background, the scanner doesn't always see the difference. We consider the problem of document binarization as a pre-processing step for optical character recognition (OCR). When scanning such a document in black-and-white, you may not be able to "drop" the background color without losing the text information as well. The background introduces "noise" that bothers the recognition or simply renders it impossible. An OCR engine does binarization internally because they work on Black \& White images. The simplest way is to calculate a threshold value and convert all pixels to white with a value above threshold value and rest of pixels convert into the black. [6] Thresholding has a huge influence on the performance of the following processes, because these are based on the data output coming from the thresholding operation. Improper thresholding can cause blotches, streaks or erasures on the document as well as confounding segmentation and recognition tasks. If someone performs optical character recognition (OCR), merges, fractures and other deformations on character shapes are the main reason of performance loss. The proposed solution uses Global thresholding as the simplest technique and the most conventional approach for binarization. A single threshold value was calculated from the global characteristics of the cropped area contain pin number.

Stroke Width Variations and Skew

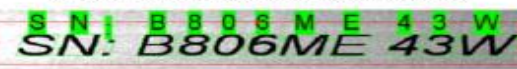

Touching Characters with Fixed Font

\section{LDัT ABC 123}

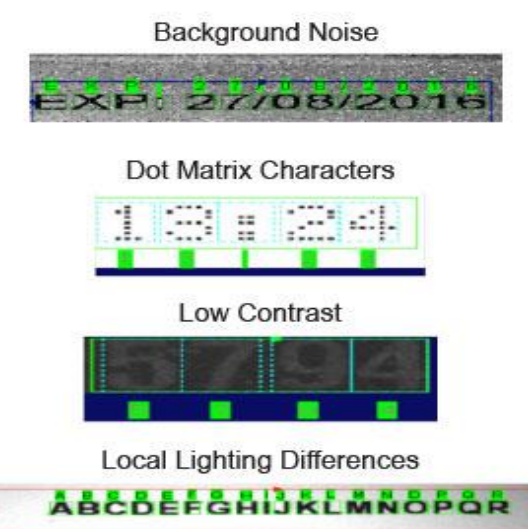

Fig 5 : OCR binarization effect 


\section{4- Noise Removal or Denoise:}

Noise decrease the readability of text from an image and reduces the accuracy of subsequent tasks of OCR (Optical character Recognition) systems. Noise removal is one of the steps in pre-processing. Noise is a random variation of color or brightness between pixels of an image and can appear in the foreground or background of an image. There are two major types of noise salt and pepper noise and Gaussian noise. [7]

\subsubsection{Segmentation}

The most common method is Optical Character Recognition (OCR) to extract text from the complex images, it is essential to segment the image and then extract the characters by using character segmentation method. Segmentation splits text from figures and graphics. When the text is segmented, it isolates characters or words. The mostly occurred problem in segmentation is it causes confusion between text and graphics in case of joined and split characters. Usually, splits and joints in the characters causes due to scanning. [8] If document is dark photocopy or if it scanned at low threshold, joints in characters will occur. And splits in characters will occur if document is light photocopy or scanned at high threshold. OCR system also gets confused during segmentation when characters are connected to graphics.

1- line segmentation. It consists of slicing a zone of interest into its different lines. Indicates the amount of added vertical spacing between the lines in our case we have only one line)

2- word and character segmentation, isolate one word from another and separate the various letters of a word.

In most cases, separating words is not that hard. There's always a distinct white space between them. (Which means that a word often includes a punctuation symbol.). Segmenting proportional characters is more difficult, because it all depends on the shape of the individual symbols The "cells" the correct technical term is "body" that each character occupies vary in surface. The unit of measure "cpi" (for "characters per inch") indicates the "pitch", the number of typographic characters that fit on each inch of a line of printed type. The measurement applies mainly to fonts that have the same width for each character (fixed fonts). Proportional fonts (with varying character widths) can only have an average cpi. Sometimes components of two adjacent characters may be touched or overlapped and this situation creates difficulties in the segmentation task. The touching or 
overlapping problem occurs frequently because of modified characters in upperzone and lower-zone.

\section{8}

Fig 6 : De-Skewed original Image (area of interest)

\begin{tabular}{|l|l|l|l|l|l|l|l|l|l|l|l|l|l|l|l|l|}
\hline 3 & 1 & 0 & 6 & 2 & & 1 & 6 & 6 & 2 & 1 & & 7 & 1 & 4 & 1 & 8 \\
\hline
\end{tabular}

Fig 7: the Segmentation effect

\subsubsection{Feature Extraction}

Feature extraction is concerned with the representation of the symbols. The feature extraction technique does not match different character patterns. Feature extraction is concerned with the representation of the symbols. Feature extraction makes note of abstract features present in a character such as intersections, open spaces, lines, etc.

Produce template images for each of the 0 to 9 digits and then detect each digit in an image and applying template matching to each digit by comparing it to the 0 to 9 templates font to obtain our digit classification. [9]

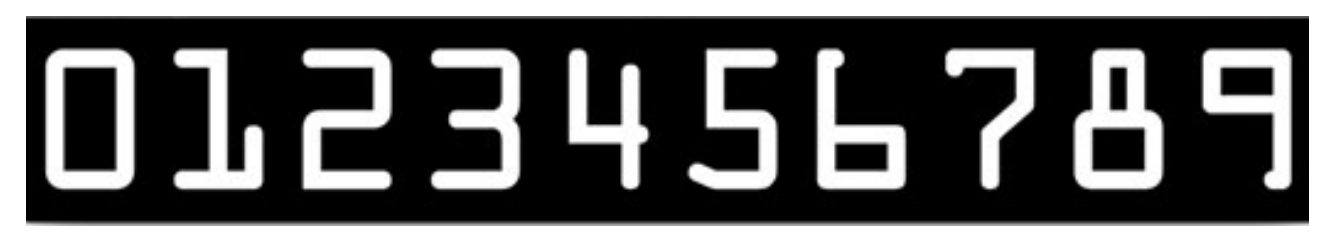

Figure 8: The OCR-A font for the digits 0-9. We will be using this font along with template matching to OCR recharge card digits in images.

In feature extraction stage, each character is represented as a feature vector, which becomes its identity. The major goal of feature extraction is to extract a set of features, which maximizes the recognition rate with the least amount of elements and to generate similar feature set for variety of instances of the same symbol. Due to the nature of handwriting with its high degree of variability and imprecision 
obtaining these features, is a difficult task. classifies the character. Feature extraction methods analyze the input document image and select a set of features that uniquely identifies and classifies the character. They are based on three types of features:

1- Statistical features- The following are the major statistical features used for character representation. Zoning- Frame of character is divided into several overlapping and non-overlapping zones. The densities of the point or some features in different regions are analyzed to form the representation. E.g. contour direction features measure the direction of the contour of the character. that are generated by dividing the image into rectangular and diagonal zones and computing histograms of chain codes in these zones. Bending point features indicate high curvature points, terminal points and fork points as shown in
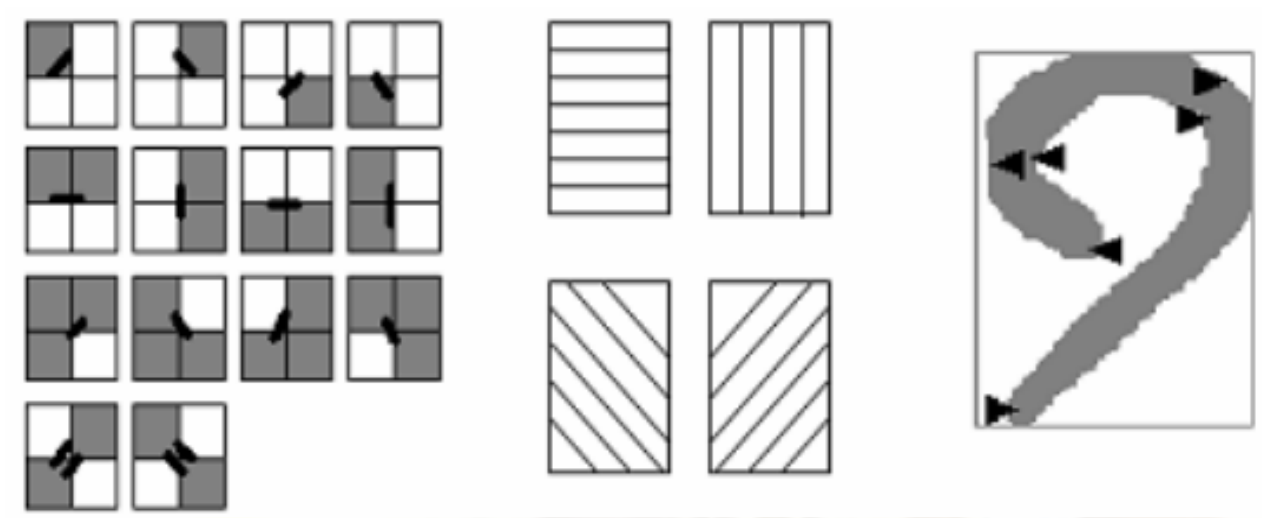

Figure 9: Contour direction and bending features with zoning.

2- Crossings and distances: It refer to the number of crossings of a contour by a line segment in a specified direction. Distance of line segment from a given boundary can be used as one of the features. A horizontal threshold can be established above, below and through the center of the script. The feature value is the count, the number of times the script crosses the threshold. [10] 

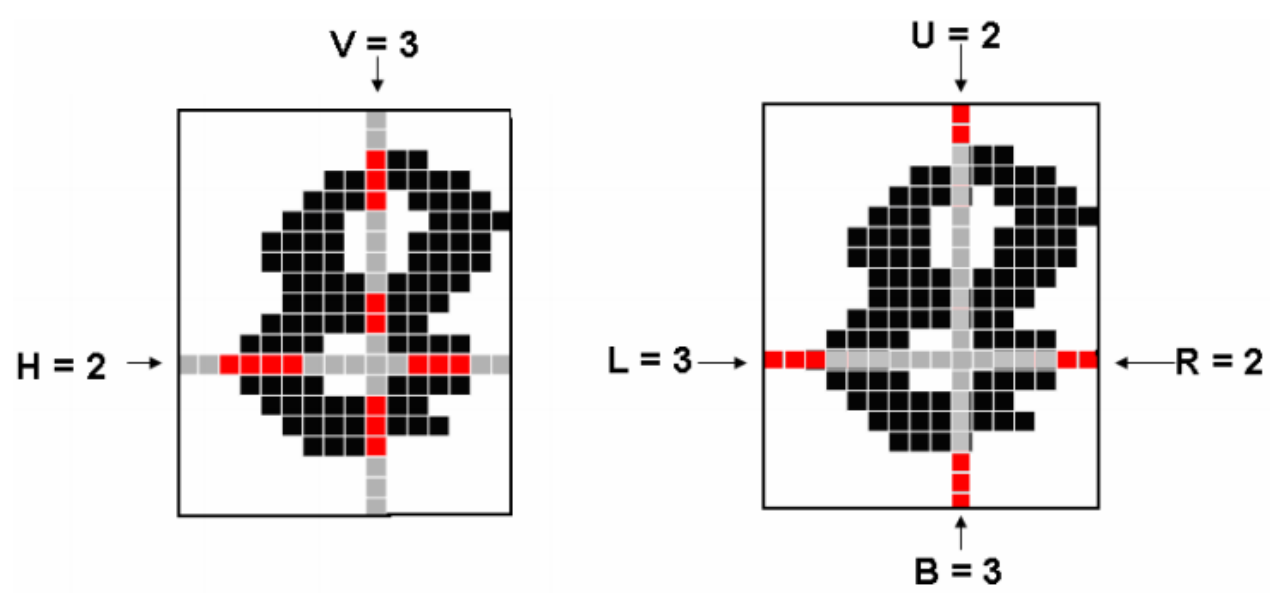

Figure 10: Example showing Crossing and Distance.

\subsection{5 post processing}

Scanning of the document and OCR introduce errors and noise. Post processing step reduce the number of errors. Post - processing corrects one sentence at the time. In post-processing we want to replace the input sequence of characters with another sequence of characters that is graphically similar and form the likeable sentence of the given language like:

- "0" or "O" or "o" or "Q" or "Ö" or "D"

- "I" or " 1 " or "!?" or "l”"

- " 8 " or "B"

- "9" or "q"

The feature differentiating classifier can apply to choose between two similar characters (e.g. between the letters " 0 " and "O"), the classifier calculates feature parameters (the slope of a line in a crucial area).

OCR output is modified by small amount of editing operations including: single character insertion, single character deletion, single character substitution, multiple character substitution ( $12 \rightarrow 21$ ), word split, word merge.[12] 


\subsubsection{Recognition}

Recognition involves converting these images to character streams representing letters of recognized words In short, recognition extracts text from images of documents. The final, classification stage is the main decision making stage of the system and uses the features extracted in the previous stage to identify the digit. We have used non parametric statistical classifier i.e. K-Nearest Neighbour for recognition purposes. The most promising recognition accuracy is achieved by using Directional Distance Distribution (DDD) features which is $95 \%$ for roman digits and $92.6 \%$ for Gurmukhi digits.[13]

\section{8

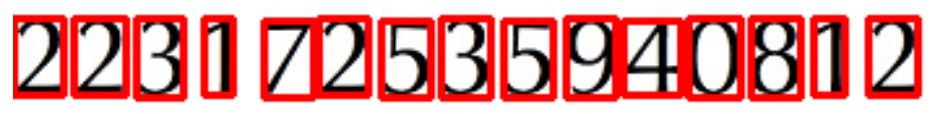

Figure 11: Performing the identification and classification

\section{Requirements and Analysis}

\subsection{Functional Requirements}

Functional requirements are the product features or its functions that must be designed directly for the users and their convenience. They define the functionality of the software, which the software engineers have to develop so that the users could easily perform their tasks up to the business requirements. So in our application the functional requirements will include the following:-

\subsection{Existence of a sim}

The application will check if there is a sim card already exists in your mobile phone or not.This is done by coding using methods and classless so no data will be required from user in this stage. The output which will be given to the user is a text saying "No Sim Inserted" in case there was no sim. In case there is a sim no text will be shown. This requirement has to go under an acceptance test to check that it is working properly so the acceptance test will be to remove sim cards and check if this text will be shown or not, and then add sim card and check if the message will disappear or not. 
Mobile Phone cards recharge using OCR .. (Applied on android platform)

\subsubsection{Number of sim cards Inserted}

The application will get number of sim cards already exists in your mobile phone. This is done by coding using methods and classless so no data will be required from user in this stage. The application will originally have a picture of two sim cards but when user first download the application and give it the permissions that it needs, it will automatically check if there are two sim cards it will leave the picture of two sim cards else if the mobile has only one sim it will remove one of the two pictures leaving only one sim picture. The acceptance test of this requirement will be to add two sim cards and check if the picture of two images will be shown or not, and then add only one sim and check that only the picture of one sim will be show.

\subsubsection{Sim-card Type}

After passing this on a feasibility study, It turns out that no way that any application can know the type of sim for example weather it is Etisalat, Vodafone , Orange or We.. The application can only know if there is as sim inserted and how many sim cards are there but coming to the type of sim it won't be able to figure that out. So in order to overcome that problem we will verify the user number. The user will enter his number and the system will send him a verification code that verifies his number. This way will help us knowing the type of user sim.

\subsubsection{Checking the validity of number}

The mobile numbers begin with 01 , consists of 11 digits and have no spaces or special characters. So, the system should check that the user number begins with 01 and that the user will enter only 11 digits and that the spaces and the special characters won't be accepted. If the user number does not start with 01 the system should send the user toast which says invalid number. If the user added space to his number, the system should not accept that and should tell him that his number is invalid. If the user added the 11 digits and is trying to add another digit the system should not allow the other digits to be added by limiting the edit text so that it can only take 11 digits and any other digit after those 11 digits won't be added.

\subsubsection{Connectivity}

In order to verify the user number, the user device should be connected to the internet otherwise the verification process will not occur. So, the system should check if user device is connected to the internet or not. If it is not connected it should send the user toast saying no internet connection to make the user pay attention that he has no internet connection. The acceptance test in this stage will be to disconnect Internet before verification process happens and see if the system will notify the user that there is no internet connection or 
not. Notice that the internet connection is only required during the verification process only because the verification code will be sent to user via Firebase server. But after that no internet connection will be needed.

\subsubsection{Change picture of sim to the logo of user company}

After verifying the user number, the system now knows which company the user belongs to, so the picture of the sim should change to the logo or something resembles his company. In order to make sure that this will work right, after verifying the user number if for example his company turns out to be Vodafone the sim picture should change to the logo of Vodafone not to any other company.

\subsubsection{Using OCR}

Recharging using camera of the system the system itself will provide its own camera. User will just click on the recharging code and OCR will output the result to the user. The

main difference that between the system's camera and the normal mobile phone camera is that the system's camera will be programmed to use OCR automatically just by clicking on the recharge code, so there is no need to capture the recharging card.

- Recharging using an existing Image After talking with the stakeholders it turns out that in some cases the user can have the picture of the recharging card stored in his gallery, so the system will provide the user with the option to pick an existing image and crop it and OCR will scan the numbers in the selected image and output the result to the user.

- Recharging by capturing the image You can have the image stored on your mobile phone or you maybe want to capture the recharging card and crop the captured image using the cropping facility provided by the system ,without having the picture saved on your gallery. Whatever method the user prefers the result will be the same. The recharging code and the quick code will be visible to the user. So, It's up to the user to choose whatever method he wants. However, If he doesn't have the image saved in his gallery which will be the most common case I think. Then he might want to go with system camera because it will work just by clicking. But if he has a saved image then of course going to the option of using OCR on existing image will suits you more. The user you will have the 2 options and can go to whatever choice you prefer. And of course, OCR should pass on acceptance test. We should make sure that OCR produce the exact desired output to the user regardless which method he chooses. 
Mobile Phone cards recharge using OCR .. (Applied on android platform)

\subsubsection{Providing the quick code}

There is a quick code which consists of 5 digits that differs from one company to another, 4 digits are added before the recharging code and the last digit is added after the recharging code. The system will add those digits after the OCR process finishes. This means that the output that will be shown to the user will be the quick code of his company + recharging code + last digit which will always be this symbol \#. So, for example My company is Etisalat the output in this case should be *556* recharging code \# and not the quick code of any other company.

\subsubsection{Recharging the code}

After OCR detect the code and the recharging code and quick code are shown to the user. The user should now be able to recharge the card just by clicking OK or submit or call option So in order to make sure that this will work right after having the quick code and the recharging code we can call them from application and check to see if the application will recharge the card successfully.

\subsubsection{Sharing the recharging card}

After talking to the stakeholders, it turns out that the users' needs a sharing option because sometimes a friend or a family member might ask them to buy a recharging code and send them the code via WhatsApp for example. So instead entering the code manually on WhatsApp app or in a normal message. It would be good idea to add a share option to the user so that in case he is sending the recharging code to someone. He can do this in a very easy way.

\section{Proposed system}

The proposed method starts by segmenting the pin number line after the binarized de-skewed charged mobile card image , horizontal and vertical projection profiles respectively. In the projection profile methods, the horizontal and vertical profiles are computed. Then each number can be segmented into individual characters by vertical projection profile. In the practical part of our application we use Tesseract Google's Optical Character Recognition (OCR). Applying Google's Tesseract resulted in low accurate digits recognition despite using Tesseract's options to recognize an image as a single text line and to OCR digits only. Note that the images background noise was removed before applying Tesseract (more on the de-noising step later in this blog). 
The proposed system examines the structure of record picture. It partitions the page into components, for example, squares of writings, tables, and pictures. The lines are separated into words and after that into characters. Once the characters have been singled out, the project contrasts them and an arrangement of example pictures. Depending on our case study we translate the scanned images of printed text into machine encoded text captured in digital form.

\subsection{Diagrams and initial design}

We use the design process in our proposed solution to help make sure that we don't miss a step or a part of the user experience that you might miss otherwise. The design process is pretty straightforward, but we find that it makes a huge difference in making sure that the final product is of the highest quality. We have used the Five UML Designs which are Use case Diagram, Activity Diagram, Sequence Diagram, State Diagram, Conceptual Class Diagram.

\subsubsection{Use Case Diagram}

We should verify everything in the application. The application is designed to recharge mobile cards using OCR and prevent the users from entering the code themselves and making mistakes. So, we will need to first make sure that the user has already sim inserted in his mobile phones. If the user doesn't have then the application should terminate. However, If the user has then I should check how many sims does he have so that the design can change accordingly. We will need to verify the user number know his company, to make the design of the application matches the company which he belongs to, and to know the quick code of his company. And

then I need to implement OCR process in order to recharge the card. I will add two ways of recharging the first one which is to use system's camera and the second one which is to use an existing image. After that I should display the result of recharging

code + quick code which will differ from one company to another.

So to sum up: -

- System will check the existence of sim

- System will check the numbers of sim 
Mobile Phone cards recharge using OCR .. (Applied on android platform)

- Design should change showing picture of one sim or two sims based on the number of sims existed

- System will verify the user number

- Design should change to something which reassembles the user company.

- System will provide 2 ways of OCR

- System will show the output of OCR process + quick code to the user.

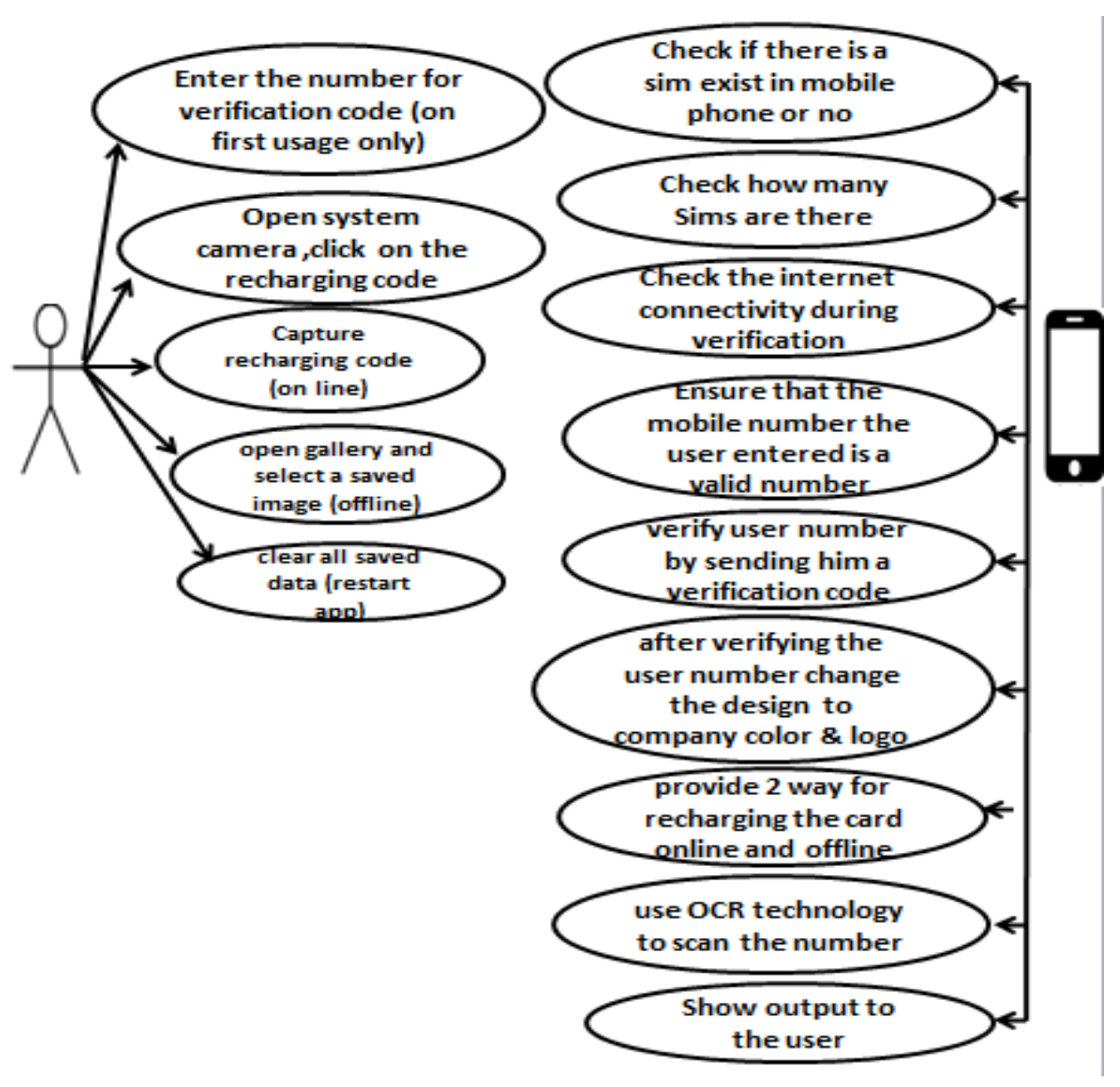

Figure 12: Use case Diagram. 


\subsubsection{Activity diagrams}

Activity diagrams describe the actual workflow behavior of a system in Information Technology. So here the activity diagram shows the workflow of my application. It shows that first the system will check existence of sim card. If there was no sim, it will leave the application. If there was it will check how many sims are there. After that the verification process will take place. The system will check if the number is valid or not if it is not valid, the system will tell the user why his number is not valid, else if it is valid. The application will send the user a verification code. After the completion of verification process the use can now recharge his card using one of 2 ways of OCR scanning either using system's camera or using an existing image. and both will show the same output. After the quick code along with the detected recharging code are shown to the user and he can now press submit in order to recharge.

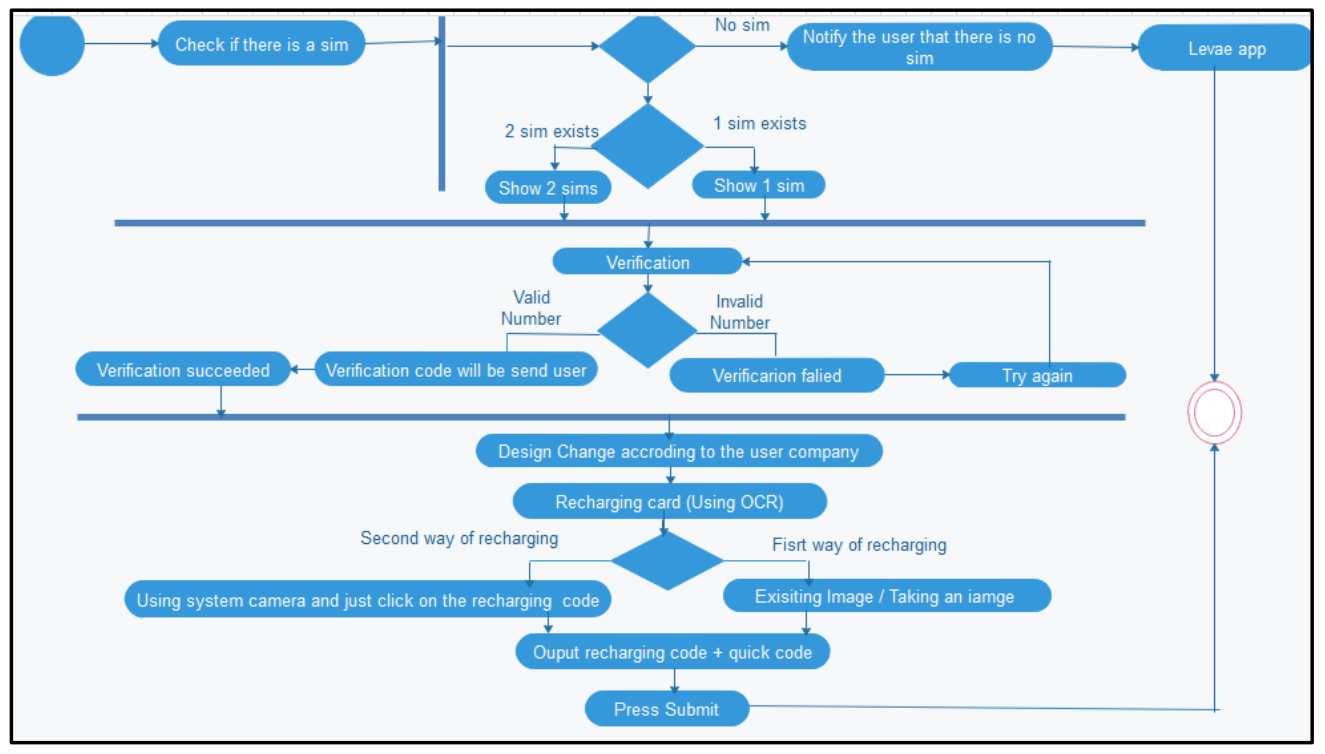

Figure 13: Activity Diagram 
Mobile Phone cards recharge using OCR .. (Applied on android platform)

\subsubsection{Sequence Diagram}

The sequence diagram shown above describes the sequence that will happen in the application the user will enter his number if it is valid, the application will send him the verification code, else if invalid it will send him a toast telling him that his number is invalid. The user then will choose the way by which he wants to recharge his card. Both ways will detect the recharging code. And the user card will be recharged.

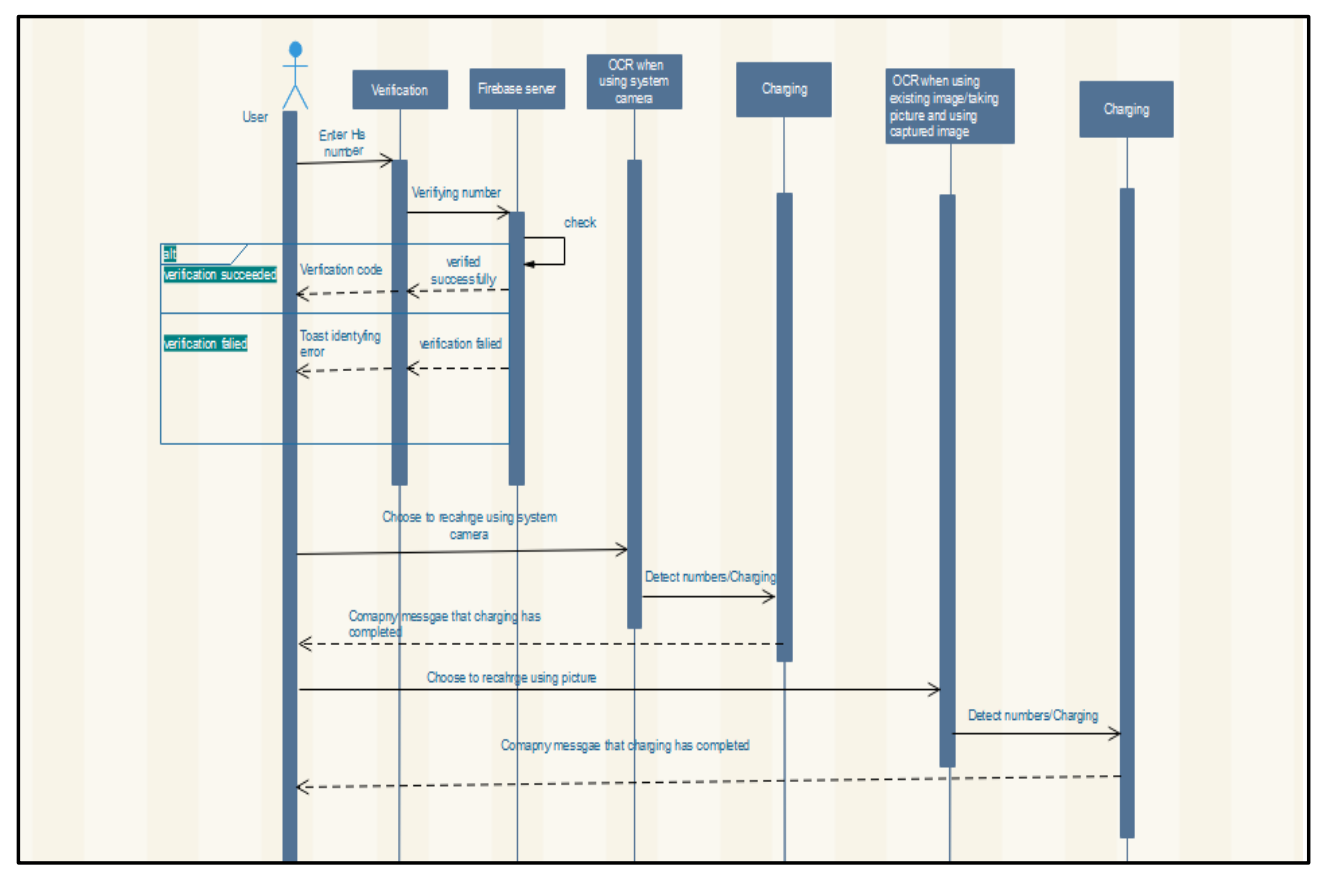

Figure 14: Sequence Diagram

\subsubsection{State Diagram}

This Diagrams Shows the different states that the system can be in first the system will check for sims after that if it will move to the verification state. when this state is completed it will move on to the OCR we have two states for OCR either using system camera or using an existing image. After that the detection will occur and the user can move to the recharging state just by clicking submit 


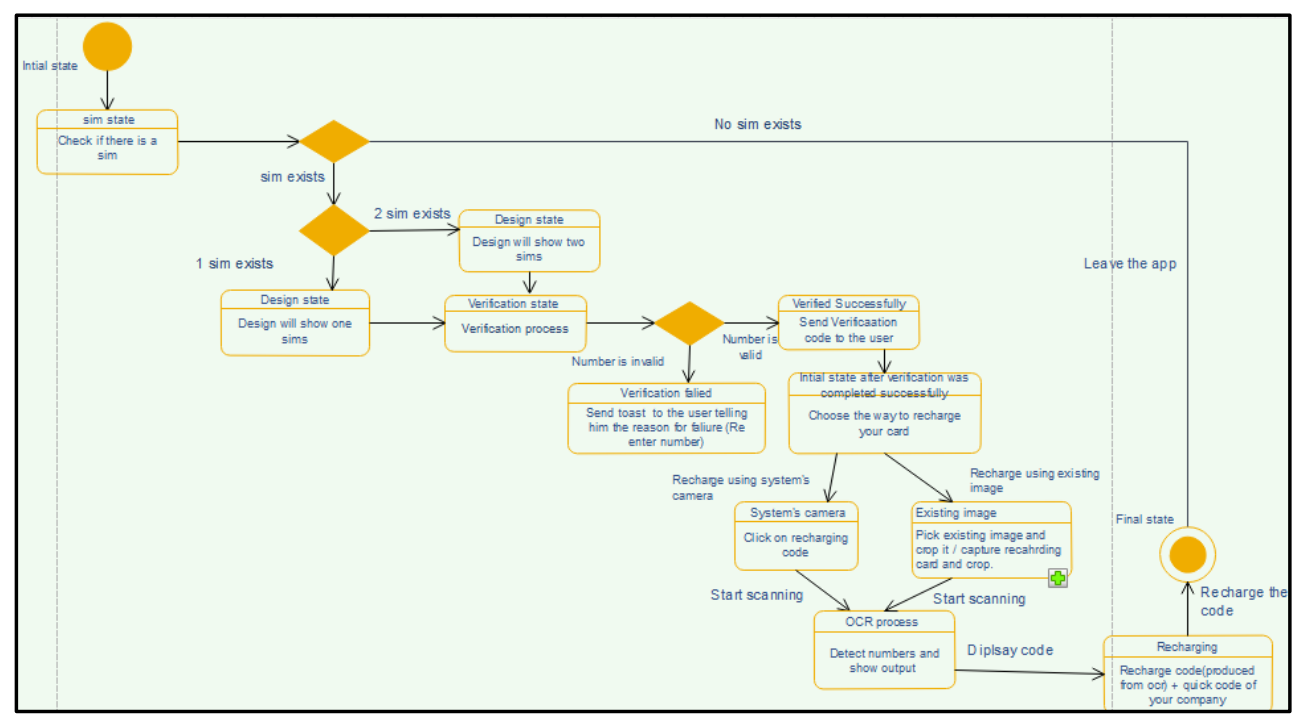

Figure 15: State Diagram

\subsubsection{Conceptual Class Diagram}

Conceptual Class diagram describes the relation between the classes in the system and how they are related to each other,And shows the attributes and the operations of eachclass.

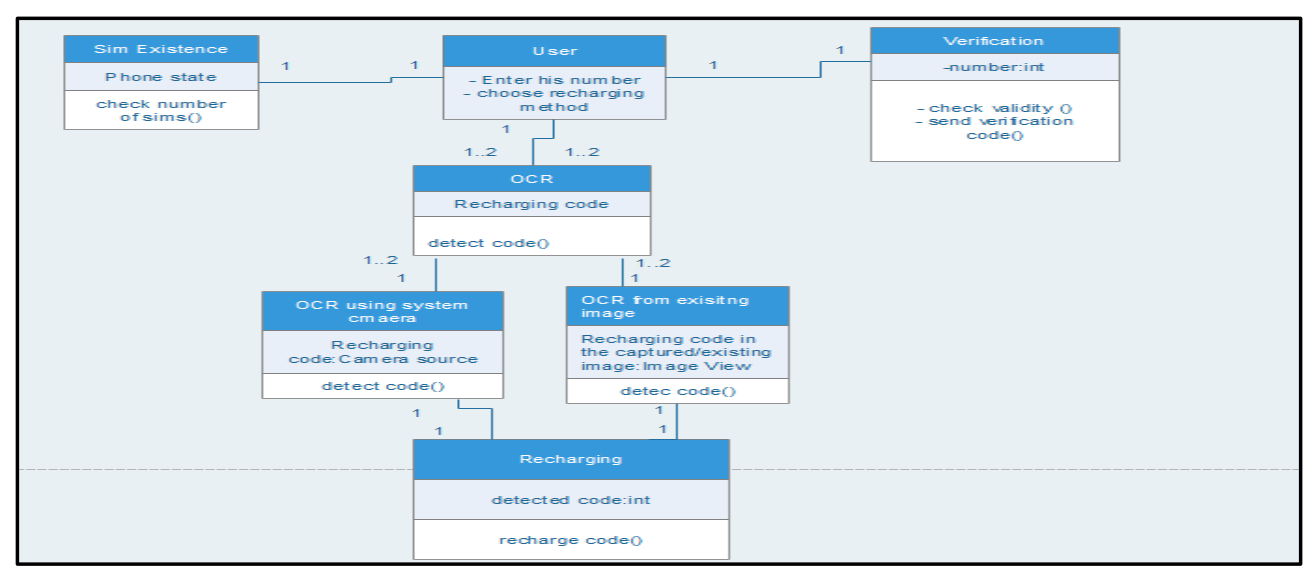

Figure 16: Conceptual Class Diagram 
Mobile Phone cards recharge using OCR .. (Applied on android platform)

\subsubsection{System Model}

The system model of the application clarifies clearly how will my application work. The application will check if sim exists or not, then the user will enter his number. If his number is invalid a toast will be sent to him. Else if it is valid then the verification

code will be sent to him via fire-base server. After that the user can choose the recharging way that he wants. After detection of code both the quick code and the recharging code will be shown to the user.

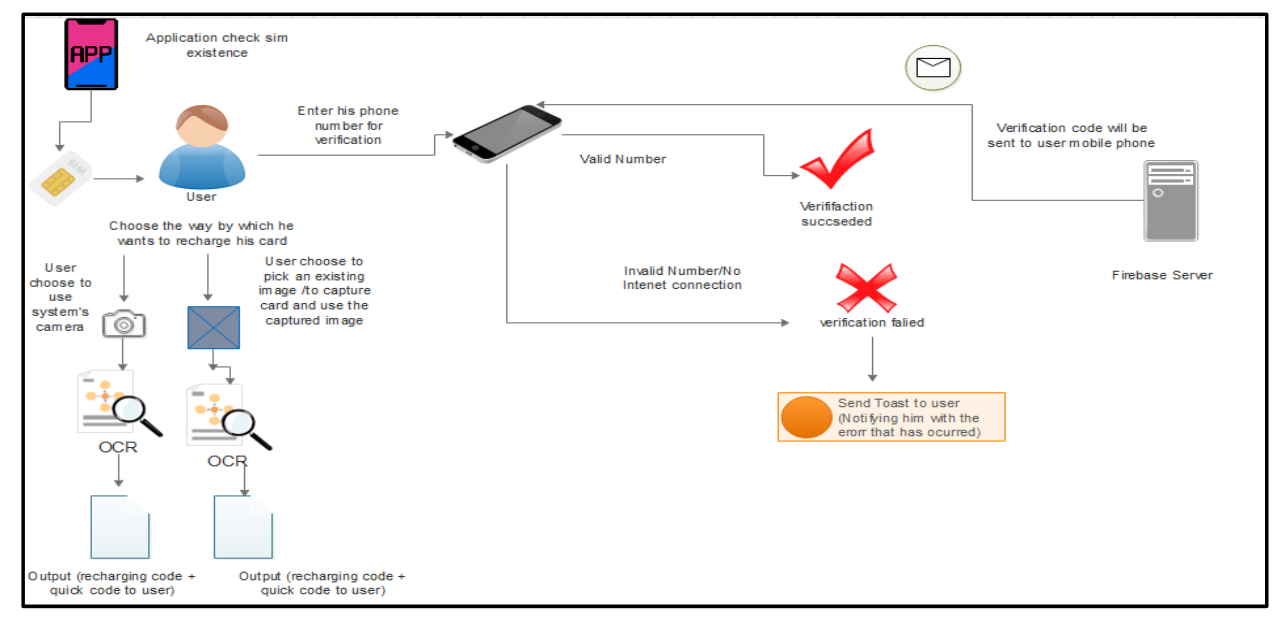

Figure 17: proposed System Model

The above figure shows that tasks that user will do. The user will first enter his number for verification, and this will happen on the first usage only. After that each time the user wants to recharge his card, he can choose which recharging ways to use.

He has 3 ways: -

- - The first way is to use system camera. In his case he will just click on the recharging code only.

- $\quad$-The second way is to pick an existing image from camera and then crop image using cropping facility provided by system.

- $\quad$-The third way is to capture the recharging card using normal camera and crop the captured image. 
Both the second and the third are nearly similar because both depend on scanning an image using OCR. They both based on having a picture.

-The user can also clear all data, which means restart the application all over again. _ As for the system: - The system will do $90 \%$ of the work. -The system will check if there is a sim inserted or not. In case there is not any sim inserted it will till the user that no

sim is inserted. -The system will check the numbers of the Sims inserted weather it is 2 or 1 and the design will change accordingly.

-The system will check your internet connectivity during verification.

- The system will also check the validity of the number. The number should start with 01 , it should consists of 11 digits, no special characters or spaces should be included

-The system will also change the design after the verification process finishes. For example if the user company is Vodafone the sim picture will change to the logo of the company and the color of the status bar and the tool bar will change also so that they can match the color of the logo.

-The system will provide 3 ways to make the user recharge his card.

-After OCR process finishes, the output will be shown to the user. The output will be the quick code which will differ from one company to another, and the recharging code which OCR produced.

\subsection{Similar application}

\begin{tabular}{|l|l|}
\hline Application & Description \\
\hline Card Scan & $\begin{array}{l}\text { Card Scan is an application which allow the user to recharge } \\
\text { his card using OCR, But the problem with that application is } \\
\text { that every time the user wants to recharge his card he has to } \\
\text { enter the quick code himself. And the design of this application } \\
\text { is very poor. }\end{array}$ \\
$\begin{array}{l}\text { https://play.google.com/store/apps/details?id=com.microblink.ocr\&hl } \\
\text { =en }\end{array}$ \\
\hline
\end{tabular}




\begin{tabular}{|l|l|}
\hline Camera & $\begin{array}{l}\text { This is another application for recharging the cards using OCR as } \\
\text { well, The problem with application is that it is similar to the Scan } \\
\text { Mobile Card } \\
\text { Card application by other means it also scans the serial number and } \\
\text { the recharging code and makes the user choose which one he wants } \\
\text { to recharge and save the other number. Moreover it also asks the } \\
\text { user to enter the prefix and the suffix on the first usage. The prefix } \\
\text { and the suffix represents the } \\
\text { quick code. } \\
\text { https://play.google.com/store/apps/details?id=jna.vision.scancard\&hl= } \\
\text { en }\end{array}$ \\
\hline JazaCredo & $\begin{array}{l}\text { This application is used constructed by Adhoc Soft Innovations Ltd } \\
\text { and is used to recharge the card of the following companies } \\
\text { Safaricom which is a mobile network operator in Kenya. It was } \\
\text { formed in 1997 as a fully owned subsidiary of Telkom Kenya. } \\
\text { Bharti Airtel which is an Indian global telecommunications } \\
\text { services company based in New Delhi, India. It operates in 20 } \\
\text { countries across South Asia and Africa. and the last one is Telkom } \\
\text { SA SOC Ltd, which is a wire line and wireless } \\
\text { telecommunications provider in South Africa, operating in more } \\
\text { than 38 countries across the African continent. The problem with } \\
\text { that application is that in it's first usage it } \\
\text { ask the user to enter his number and does not send him any } \\
\text { verification code to make sure that this is his number, so the } \\
\text { user can enter any valid number even if this number is not his } \\
\text { number and then proceed with the application. There is nothing } \\
\text { to ensure that this number is the user number himself. } \\
\text { https://play.google.com/store/apps/details?id=com.adhocsoft.jazacr } \\
\text { edo\&hl=en }\end{array}$ \\
\hline
\end{tabular}

\section{Common disadvantages}

1) There is no sort of sim detection All the previous application does not detect if there was a sim already inserted or not, So they do the OCR process even if there was no sim inserted. If the user has two sims inserted in his mobile phone the application will do the OCR process and after the card is scanned and the user click ok. A Dialog box is shown to the user asking him which sim he wants to recharge. And this happens in normal dial pad. After you enter your 
recharging code manually on the dial pad and press the call button the system asks you which sim you want to recharge.

2) They only provide one way of OCR scanning Does not provide the user with the option to pick an existing image of recharging card or to capture the whole card and then crop the recharging card and scan the code .And providing more than one way to the user is important because now It will give the user to choose the way he prefer in order to recharge his card

3) User has to enter the quick code himself. The user has to enter the quick code himself because the previous application does not have any sort of verification process and so they cannot know the user company and hence they won't be able

to know the quick code so they ask the user to enter the quick code every time

4) Design of all the previous applications is very poor. The Design of interfaces is one of most important things that attract the users to your application, so the design should be user friendly, it should attract the user, should be unambiguous and it should be an interactive design and this is not the case in the previous applications.

\section{Methods}

In this part we will demonstrate how we think in ways that allow finding efficient solutions to problems we face through theory and practical software Development.

\begin{tabular}{|l|l|}
\hline General problems & Technical Solution \\
\hline $\begin{array}{l}\text { Different mobile Network Operator } \\
\text { (MNO) with different recharge code } \\
\text { consist of the recharging 15 digits } \\
\text { and to the quick code which consists } \\
\text { of 5 digits }\end{array}$ & $\begin{array}{l}\text { The mobile application will make } \\
\text { the user recharge their mobile phone } \\
\text { automatically without having to } \\
\text { enter the charging code themselves. } \\
\text { All they have to do is to the click on } \\
\text { the recharging code and it will be } \\
\text { converted in to editable numbers } \\
\text { using optical character recognition } \\
\text { to the correct Mobile Network } \\
\text { Operator (MNO) and we ensure that } \\
\text { the code will be inserted correctly } \\
\text { from the first time because the user } \\
\text { will not insert any digit himself. }\end{array}$ \\
\hline Use case problems & Technical Solution \\
\hline
\end{tabular}




\begin{tabular}{|c|c|}
\hline $\begin{array}{l}\text { The first problem is that the } \\
\text { application will need to know the type } \\
\text { of the user's sim or what Mobile } \\
\text { Network Operator (MNO) he belongs } \\
\text { to in order to be able to automatically } \\
\text { enter the quick code which differs } \\
\text { from one mobile network carrier to } \\
\text { another. }\end{array}$ & $\begin{array}{l}\text { we will verify the number of user. } \\
\text { The user will enter his number on } \\
\text { the very first usage of the } \\
\text { application and I will check it and } \\
\text { according to his number the } \\
\text { application will be able to know his } \\
\text { Mobile Network Operator (MNO) } \\
\text { and so determine the type of the sim } \\
\text { and so it will be able to know the } \\
\text { quick code of his mobile network } \\
\text { carrier. }\end{array}$ \\
\hline $\begin{array}{l}\text { The small and bad clearly for the } \\
\text { charging numbers decrease The OCR } \\
\text { accuracy }\end{array}$ & $\begin{array}{l}\text { There are plenty of APIs that } \\
\text { can be used to ensure Optical } \\
\text { character recognition accuracy } \\
\text { like Zxing, Tesseract ,etc. But the } \\
\text { best of them all is Google } \\
\text { Vision API which was first released } \\
\text { on } 2015 \text { because it provides } \\
\text { very high degree of accuracy, } \\
\text { powerful and reliable Optical } \\
\text { character recognition (OCR) library } \\
\text { and work on most of the android } \\
\text { device. }\end{array}$ \\
\hline
\end{tabular}

The proposed application will make the user choose the way by which he wants to recharge the card. The application will not be like normal dial-pad which we normally use in order to recharge the cards. The application will just focus on recharging the cards in an accurate and efficient using OCR. This is our target.

\section{Results and Discussion}

This section incorporates the result we have found by testing our application on various conditions. We have considered various criteria's for ascertaining the outcome, which is extraordinary light conditions, for various matured individuals, in various gadgets for various administrators

After searching and studying the disadvantages of the previous applications. The proposed application has the following features. 
It provides two ways of OCR scanning not just one. And the user will choose which method he wants to choose. And so, I am giving the user more than one option in order to recharge his card not just one. Moreover, it will detect if there was a sim existed or not. Because if there was no sim detected then it will till the user that there is no sim in his mobile phone and the scanning process won't even take place from the very first beginning. In case there was more 1 sim it will show that there is one sim and if there are two sims it will show two sims and the user can choose which sim he wants to recharge. And this will make the recharging process done without asking the user at the end which sim is this recharging code belongs to like what happens in the previous application. Furthermore, It will provide a verification process to know the user sim type and so the design can change accordingly to something that reflects the user company and something that is pleasant to the user. The verification process will also determine the quick code of the company which the user belongs to. It will also add the quick code to the user according to his company. So, the user will not add the quick code himself. The design will be pleasant to the user. And the application will be user friendly and unambiguous and easy for normal user to understand and interact with.

There are four mobile service providers in Egypt, namely Vodafone, Orange, Etisalat and WE. We have collected 10 pre-paid recharge cards for each Mobile Network Operator (MNO) for testing the accuracy rate of our applications in different condition Sun light, Flash light and Normal light. We have calculated the accuracy rate in terms of the recognized the total correct pin numbers detected by our proposed solution by from the total pin numbers

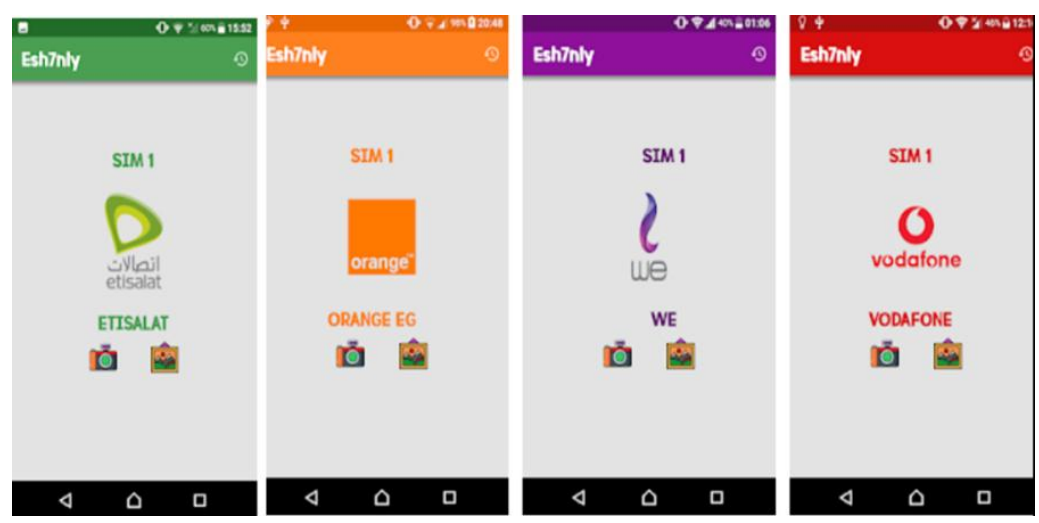

Figure 13: changed layout design automatic according to service operator SIM card 
Mobile Phone cards recharge using OCR .. (Applied on android platform)

\begin{tabular}{|c|c|c|c|c|c|}
\hline & Operator & $\begin{array}{l}\text { Total Pin } \\
\text { Numbers }\end{array}$ & $\begin{array}{l}\text { Recognized } \\
\text { Pin } \\
\text { Numbers }\end{array}$ & $\begin{array}{l}\text { Accuracy } \\
\text { Rate\% }\end{array}$ & DETECTED \\
\hline $\begin{array}{l}\text { Vodafon } \\
\mathrm{e}\end{array}$ & CARD1 & 16 & 16 & 100 & YES \\
\hline $\begin{array}{l}\text { Vodafon } \\
\text { e }\end{array}$ & CARD2 & 16 & 16 & 100 & YES \\
\hline $\begin{array}{l}\text { Vodafon } \\
\text { e }\end{array}$ & CARD3 & 16 & 16 & 100 & YES \\
\hline $\begin{array}{l}\text { Vodafon } \\
\text { e }\end{array}$ & CARD4 & 16 & 16 & 100 & YES \\
\hline $\begin{array}{l}\text { Vodafon } \\
\text { e }\end{array}$ & CARD5 & 16 & 16 & 100 & YES \\
\hline $\begin{array}{l}\text { Vodafon } \\
\text { e }\end{array}$ & CARD6 & 16 & 16 & 100 & YES \\
\hline $\begin{array}{l}\text { Vodafon } \\
\mathrm{e}\end{array}$ & CARD7 & 16 & 16 & 100 & YES \\
\hline $\begin{array}{l}\text { Vodafon } \\
\text { e }\end{array}$ & CARD8 & 16 & 16 & 100 & YES \\
\hline $\begin{array}{l}\text { Vodafon } \\
\text { e } \\
\end{array}$ & CARD9 & 16 & 14 & 87.5 & NO \\
\hline $\begin{array}{l}\text { Vodafon } \\
\text { e }\end{array}$ & CARD10 & 16 & 16 & 100 & YES \\
\hline Orange & CARD1 & 15 & 15 & 100 & YES \\
\hline Orange & CARD2 & 15 & 15 & 100 & YES \\
\hline Orange & CARD3 & 15 & 15 & 100 & YES \\
\hline Orange & CARD4 & 15 & 15 & 100 & YES \\
\hline Orange & CARD5 & 15 & 15 & 100 & YES \\
\hline Orange & CARD6 & 15 & 15 & 100 & YES \\
\hline Orange & CARD7 & 15 & 15 & 100 & YES \\
\hline Orange & CARD8 & 15 & 15 & 100 & YES \\
\hline Orange & CARD9 & 15 & 13 & 86.666667 & NO \\
\hline Orange & CARD10 & 15 & 15 & 100 & YES \\
\hline
\end{tabular}




\begin{tabular}{|c|c|c|c|c|c|}
\hline Etisalat & CARD1 & 15 & 15 & 100 & YES \\
\hline Etisalat & CARD2 & 15 & 15 & 100 & YES \\
\hline Etisalat & CARD3 & 15 & 15 & 100 & YES \\
\hline Etisalat & CARD4 & 15 & 15 & 100 & YES \\
\hline Etisalat & CARD5 & 15 & 11 & 73.333333 & NO \\
\hline Etisalat & CARD6 & 15 & 15 & 100 & YES \\
\hline Etisalat & CARD7 & 15 & 15 & 100 & YES \\
\hline Etisalat & CARD8 & 15 & 15 & 100 & YES \\
\hline Etisalat & CARD9 & 15 & 15 & 100 & YES \\
\hline Etisalat & CARD10 & 15 & 15 & 100 & YES \\
\hline WE & CARD1 & 15 & 15 & 100 & YES \\
\hline WE & CARD2 & 15 & 15 & 100 & YES \\
\hline WE & CARD3 & 15 & 15 & 100 & YES \\
\hline WE & CARD4 & 15 & 15 & 100 & YES \\
\hline WE & CARD5 & 15 & 15 & 100 & YES \\
\hline WE & CARD6 & 15 & 15 & 100 & YES \\
\hline WE & CARD7 & 15 & 14 & $\mathbf{9 3 . 3 3 3 3 3 3}$ & NO \\
\hline WE & CARD8 & 15 & 15 & 100 & YES \\
\hline WE & CARD9 & 15 & 15 & 100 & YES \\
\hline \multirow[t]{2}{*}{ WE } & CARD10 & 15 & 15 & 100 & YES \\
\hline & $\begin{array}{l}\text { Average } \\
\text { Accuracy }\end{array}$ & 610 & 601 & 0.9852459 & \\
\hline
\end{tabular}




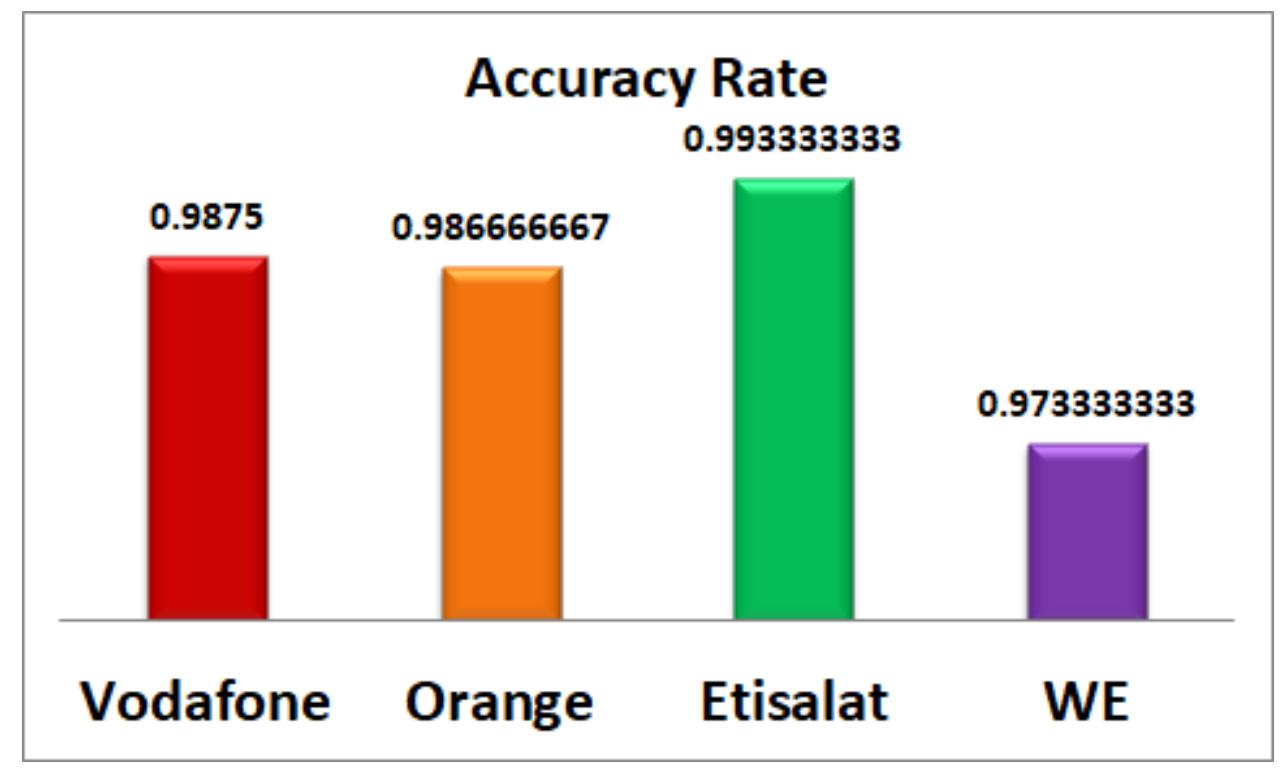

Figure 14: proposed accuracy rate

A use case diagram at its simplest is a representation of a user's interaction with the system that shows the relationship between the user and the different use cases in which the user is involved.

\section{Conclusion}

To sum up the idea We use the OCR technology and develop an application to enable the users recharge their mobile card automatically without having to enter the recharging code themselves. So, we developed OCR mobile phone cards recharge application. Input will be the recharging code then OCR will process this code and convert into editable numbers and show the output to the user. The deliverables of the application will be the quick code which differs from one company to another and the recharging code produced rom OCR process. The application is now published on the play store under the name Esh7nly. The experiment result shows recognition rate is $98.5 \%$ Further work is initiated for multiple font and size characters and hand written character recognition. 

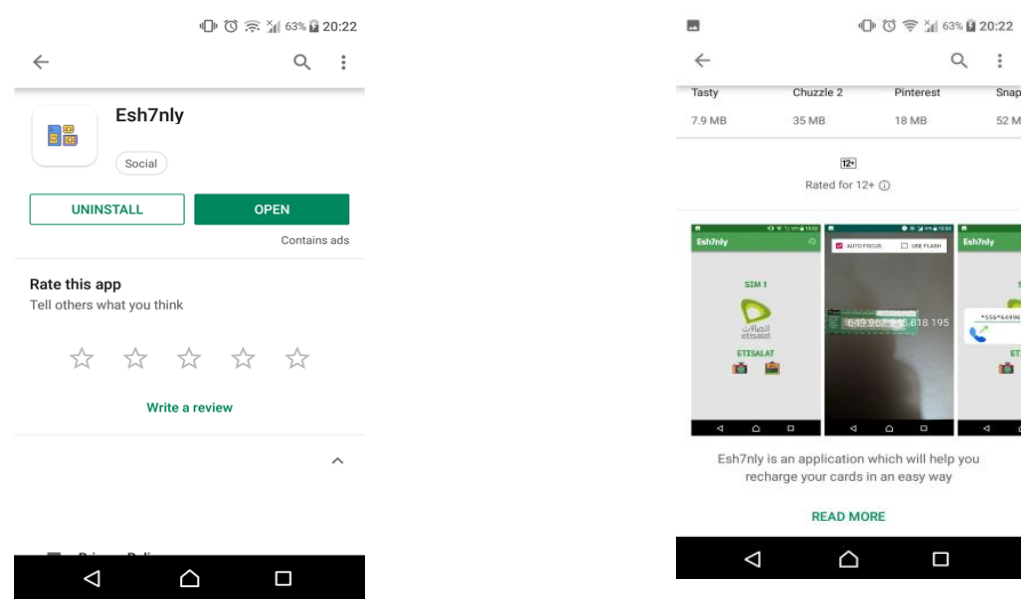

Figure 11: The proposed application Esh7nly on the play store

\section{ACKNOWLEDGMENTS}

Practical Application was done at Laboratory of Image Processing, El-Shourouk Academy, Higher Institute for Computer \& Information Technology, Egypt. The financial support by sherouk academy for Technology and Innovation is gratefully acknowledged.

\section{References}

[1] https://www.statista.com/statistics/274774/forecast-of-mobile-phone-usersworldwide.

[2] "Manage your account". AT\&T Residential Wireless Support. AT\&T. Retrieved 11 May 2013

[3]. Kumar R., Singh A., "Challenges in Segmentation of Text in Handwritten Gurmukhi Script" Proceedings in BAIP 2010, CCIS 70, Springer-Verlag Berlin Heidelberg, pp. 388-392, 2010

[4]. Binny Thakral, Manoj Kumar, "Devanagari Handwritten Text Segmentation for Overlapping and Conjunct Characters- A Proficient Technique". 978-14799-6896-1,IEEE 2014. 
Mobile Phone cards recharge using OCR .. (Applied on android platform)

[5]. Naunita, Taneja A., Chawla M., "Segmentation of Touching Characters in Handwritten Gurumukhi Script", International Journal of Engineering Sciences, Vol. 3, pp. 90-94, 2014.

[6]. Kumar R., Singh A., "Algorithm to Detect and Segment Gurmukhi Handwritten Text into Lines, Words and Characters", IACSIT International Journal of Engineering and Technology, Vol.3, No.4, 2011.

[7]. Kumar R., Singh A., "Detection and Segmentation of Lines and Words in Gurmukhi Handwritten Text" Institute of Electrical and Electronics Engineers (IEEE), pp. 353-356, 2010.

[8]. Bansal G., Sharma D., "Isolated Handwritten Words Segmentation Techniques in Gurmukhi Script", International Journal of Computer Applications, Vol. 1, No. 24, pp. 104-111, 2010.

[9]. Garg N.K., Kaur L., Jindal M.K. "The segmentation of half characters in Handwritten Hindi Text”, SpringerVerlag Berlin Heidelberg, pp. 48-53, 2011.

[10]. Kumar D., Koshti, Govilkar S., "Segmentation of Touching Characters in Handwritten Devanagri Script", International Journal of Computer Science and its Applications, Vol. 2, Issue 2, pp. 83-87.

[11]. Kumar M., Jindal M.K., Sharma R.K., "Segmentation of Isolated and Touching Characters in Offline Handwritten Gurmukhi Script Recognition", International Journal Information Technology and Computer Science, pp. 58- 63, Feb, 2014.

[12]. Mangla P., Kaur H., “An End Detection Algorithm for segmentation of Broken and Touching characters in Gurumukhi Word", Handwritten Institute of Electrical and Electronics Engineers (IEEE) , pp.1-4, 2014.

[13]. Mehta B., Rani S., "Segmentation of Broken Characters of handwritten Gurmukhi Script", International Journal of Engineering Sciences, Vol. 3, pp. 95-105, 2014. 